\title{
Efficacy of Nilotinib versus Imatinib in Philadelphia Positive Patients with Chronic Myeloid Leukemia in Early Chronic Phase Who Have a Warning Molecular Response to Imatinib
}

\author{
Amen Hamdy Zaky Aly¹, Ahmed EL Sayed Mohammed Khalifa², \\ Esraa Abdallah Abdelkariem Gadallah ${ }^{3}$ \\ ${ }^{1}$ Department of Medical Oncology, South Egypt Cancer Institute, Assuit University, Assiut, Egypt \\ ${ }^{2}$ Department of Clinical Oncology, Faculty of Medicine, Sohag University, Sohag, Egypt \\ ${ }^{3}$ Department of Medical Oncology, Sohag Cancer Center, Sohag, Egypt \\ Email: esraaabdallah25@gmail.com
}

How to cite this paper: Aly, A.H.Z., Khalifa, A.S.M. and Gadallah, E.A.A. (2018) Efficacy of Nilotinib versus Imatinib in Philadelphia Positive Patients with Chronic Myeloid Leukemia in Early Chronic Phase Who Have a Warning Molecular Response to Imatinib. Journal of Cancer Therapy, 9, 883-897.

https://doi.org/10.4236/jct.2018.911073

Received: September 18, 2018

Accepted: November 13, 2018

Published: November 16, 2018

Copyright (c) 2018 by authors and Scientific Research Publishing Inc. This work is licensed under the Creative Commons Attribution International License (CC BY 4.0).

http://creativecommons.org/licenses/by/4.0/

\begin{abstract}
Background and Objectives: Chronic myeloid leukemia (CML) accounts for approximately $15 \%$ of newly diagnosed cases of leukemia in adults. In this study, the efficacy of nilotinib at $400 \mathrm{mg}$ BID is compared with imatinib at $400 \mathrm{mg}$ BID in CML patients with suboptimal molecular response after at least 12 months of daily dose $400 \mathrm{mg}$ of imatinib therapy. Patients and Methods: This study included a total number of 50 patients, divided into two groups ( 25 patients each). The first group (Group I): Patients received imatinib at $400 \mathrm{mg} \mathrm{BID}$, second group (Group II): Patients had a suboptimal molecular response to imatinib and received nilotinib at $400 \mathrm{mg}$ BID in early chronic phase. During the two years period of data collection, the primary end included median survival. The secondary end included response rate, type of response, duration of response and progression free survival. Also side effects were recorded. Patients were followed up every month by complete and differential blood counts, liver function test, renal function test and (PCR) every three months for two year. Results: Nilotinib group had significantly higher frequency of major molecular response (MMR) where 23 (92\%) patients achieved it while only $16(64 \%)$ patients in Imatinib group achieved MMR $(P=0.01)$. Nilotinib had better toxicities profile than Imatinib. Conclusion: Both Nilotinib and high dose Imatinib achieved response in CML patients with suboptimal response with rapid and deeper molecular response, better survival outcomes and less side effects in nilotinib.
\end{abstract}




\section{Keywords}

Nilotinib, Imatinib, Philadelphia Positive Chronic Myeloid Leukemia

Patients, Warning Molecular Response

\section{Introduction}

Chronic myeloid leukemia (CML) is a myeloproliferative neoplasm with an incidence of $1-2$ cases per 100,000 adults. It accounts for approximately 15\% of newly diagnosed cases of leukemia in adults [1].

In 2002, the approval of the BCR-ABL tyrosine kinase inhibitor (TKI) imatinib mesylate for the first line treatment of chronic myeloid leukemia revolutionized therapeutic goals and led to the introduction of novel monitoring schemes and response definitions [2].

However, many patients treated with imatinib do not meet the molecular response targets designated by the National Comprehensive Cancer Network (NCCN) and the European Leukemia Net [3].

The response to TKI can be classified as optimal and failure. The term suboptimal may be better replaced by the term "warning", meaning that between optimal and failure, there is a gray zone where the response must be monitored more carefully and that the patient may be eligible for potentially better treatments. The choice of the treatment, particularly the decision of moving from one treatment to another, strongly depends on the response to treatment, particularly on the degree of the cytogenetic response $(\mathrm{CgR})$, and on the detection of BCR-ABL KD point mutations [4].

Among patients with $\mathrm{CCyR}$ at 12 months in the International Randomized Study of Interferon Versus STI571 (IRIS) trial of frontline imatinib, $>30 \%$ of patients did not achieve MMR at 12 months [5].

Nilotinib is a highly selective $B C R-A B L 1$ TKI approved for the treatment of patients with newly diagnosed CML-CP or imatinib-resistant or imatinib-intolerant CML-CP or CML-AP. With 5 years of follow up in the Evaluating Nilotinib Efficacy and Safety in Clinical Trials-Newly Diagnosed Patients (ENESTnd) study, frontline nilotinib resulted in higher response rates and a lower risk of disease progression versus frontline imatinib for patients with $\mathrm{Ph}+\mathrm{CML}-\mathrm{CP}[6]$.

The ELN considers patients with a response level equivalent to CCyR without MMR ( $B C R-A B L I I S>0.1 \%-1 \%$ ) at 12 months following initiation of frontline TKI therapy to be in a "warning zone" indicating that they may be at risk for treatment failure [7].

In this study, we compared the efficacy of nilotinib at $400 \mathrm{mg}$ BID with imatinib at $400 \mathrm{mg}$ BID in suboptimal molecular response patients after at least 12 months of daily dose $400 \mathrm{mg}$ of imatinib therapy and we excluded patients who started late chronic phase Imatinib therapy more than 6 months, patients who had blastic or accelerated crises, patients with impaired cardiac function, im- 
paired gastrointestinal (GI) function or GI disease, hypersensitivity to Nilotinib or intolerance to Imatinib, severe or uncontrolled medical conditions i.e. (uncontrolled diabetes, acute or chronic liver disease, pancreatic, or severe renal disease unrelated to tumor, active or uncontrolled infection).

\section{Patients and Methods}

This comparative prospective study was carried out after approval of the local ethics committee, where we included a total number of 50 patients, fulfilling the after-coming inclusion and exclusion criteria, who divided into two groups. The first group (Group I): included 25 patients who received imatinib at $400 \mathrm{mg}$ BID, second group (Group II): included 25 patients and they received nilotinib at 400 mg BID in early chronic phase that had a suboptimal molecular response to imatinib.

Patients included were male or female patients with a performance status (ECOG): 0 - 2, diagnosis of phladelphia positive chronic myeloid leukemia in chronic phase, patients with suboptimal molecular response defined as patients not achieving a complete cytogenic response at 12 months and must maintain complete cytogenic response until study entry ( $0 \%$ pheladephia +ve chromosome). Patients should receive at least 18 months and up to 24 months of treatment with Imatinib as first line therapy at a dose of $400 \mathrm{mg}$ daily without achieving a major molecular response $(<0.1 \%$ IS of Pcr-Apl transcript by RQ-PCR), no renal or hepatic impairment.

Exclusion criteria included late chronic phase patients who started Imatinib more than 6 months after diagnosis, prior accelerated phase or blast phase CML, hypersensitivity to Nilotinib or intolerance to Imatinib, previous treatment with interferon or any other tyrosine kinase inhibitor except Imatinib, patients treated with Imatinib more than $400 \mathrm{mg} /$ day who achieved prior major molecular or complete cytogenic response on Imatinib and lost response to enter the study, impaired cardiac or GIT functions, severe or uncontrolled medical conditions (uncontrolled diabetes mellitus, uncontrolled acute or chronic liver disease, pancreatic or severe renal disease unrelated to tumor, or uncontrolled infection), any other malignancy that is clinically significant or requiring medical interventions.

During the two years period of data collection, the primary end point included median survival. The secondary end points included response rate, type of response, duration of response and progression free survival.

Also side effects including nausea, vomiting, diarrhea, fatigue, myalgia and Q-T interval abnormalities were recorded.

\section{Patients' follow up:}

Patients followed up for two year by complete blood count with differential count every month, liver function test, renal function test and (PCR) every three months.

A data entry file, using EXCEL 2016 program, was prepared. Data were processed and analyzed using SPSS ver. 20 . The frequencies, percentages, mean 
and standard deviation were computed. Independent samples t-test was used to compare quantitative data between the two groups. Chi-square test was used to compare qualitative data between two groups. $P$-value of $<0.05$ was considered as significant.

\section{Results}

During the two years of follow up period of the study, 25 patients were enrolled in Nilotinib group and 25 patients were enrolled Imatinib group. No deaths or patients losses during study period.

Demographic data were equally distributed among both groups.

There was male predominance in both groups, but this was not a significant difference between the two groups $(P=0.09)$ (Table 1$)$.

Outcome of therapy in each group:

- At the $12^{\text {th }}$ month therapy

Regarding the major molecular response (MMR) at the $12^{\text {th }}$ month of therapy, Nilotinib group had significantly higher frequency of MMR where 20 (80\%) patients achieved it while only18 (72\%) patients in Imatinib group achieved MMR $(P=0.04)$ (Table 2, Figure 1$)$.

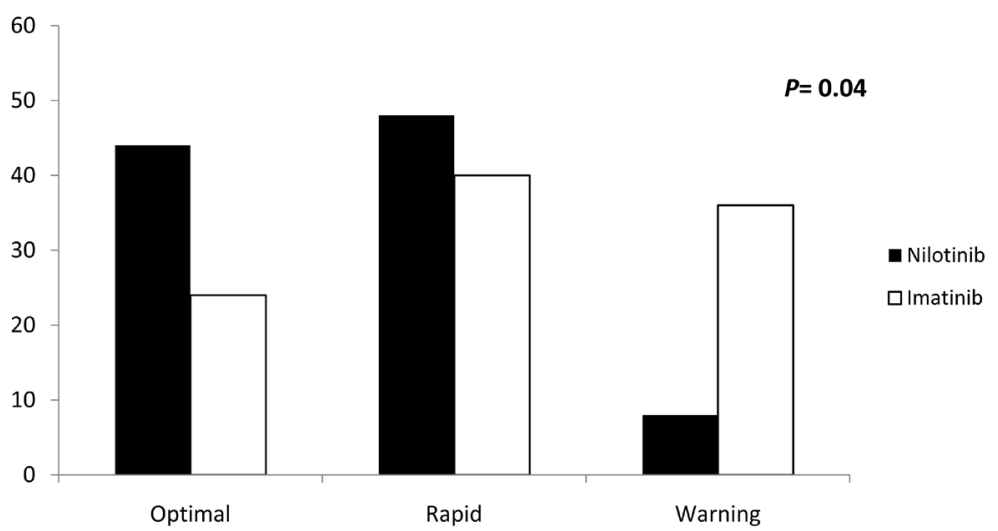

Figure 1. Type of major molecular response. Data was expressed in form of percentage.

Table 1. Demographic data of both groups.

\begin{tabular}{cccc}
\hline Variables & Nilotinib group $(\mathbf{n}=\mathbf{2 5})$ & Imatinib group $(\mathbf{n}=\mathbf{2 5})$ & $P$ value \\
\hline Age (years) & $47.24 \pm 10.43$ & $44.04 \pm 9.81$ & \\
$<40$ years & $6(24 \%)$ & $12(48 \%)$ & 0.32 \\
$>40$ years & $19(76 \%)$ & $13(52 \%)$ & \\
Sex & & & 0.09 \\
Male & $21(84)$ & $19(76)$ & \\
Female & $4(16)$ & $6(24)$ & \\
Residence & & $12(48 \%)$ & 0.65 \\
Urban & $10(40 \%)$ & $13(52 \%)$ & \\
Rural & $15(60 \%)$ & & \\
\hline
\end{tabular}

Data was expressed in form of frequency (percentage) or mean \pm SD. $P$ value was significant if $<0.05$. n: number. 
Table 2. Response of therapy in each group.

\begin{tabular}{|c|c|c|c|}
\hline Outcome & $\begin{array}{l}\text { Nilotinib group } \\
\quad(\mathrm{n}=25)\end{array}$ & $\begin{array}{l}\text { Imatinib group } \\
\quad(\mathrm{n}=25)\end{array}$ & $P$ value \\
\hline \multicolumn{4}{|l|}{ At the $12^{\text {th }}$ month } \\
\hline Major molecular response & $20(80)$ & $18(72)$ & $0.04^{\star}$ \\
\hline Type of response & & & $0.01^{*}$ \\
\hline Optimal & $12(48)$ & $11(44)$ & \\
\hline Early moleculr & $8(32)$ & $7(28)$ & \\
\hline Warning & $5(20)$ & $7(28)$ & \\
\hline \multicolumn{4}{|l|}{ At the $18^{\text {th }}$ month } \\
\hline Major molecular response & $22(88)$ & $16(64)$ & $0.02^{*}$ \\
\hline Type of response & & & $0.02^{*}$ \\
\hline Optimal & $12(48)$ & $9(36)$ & \\
\hline Earlymolecular & $10(40)$ & $7(28)$ & \\
\hline Warning & $3(6)$ & $9(36)$ & \\
\hline \multicolumn{4}{|l|}{ At the end of the study } \\
\hline Major molecular response & & $16(64)$ & $0.01^{*}$ \\
\hline Type of response & & & $0.04^{*}$ \\
\hline Optimal & & $6(24)$ & \\
\hline Early molecular & & $10(40)$ & \\
\hline Warning & & $9(36)$ & \\
\hline
\end{tabular}

Data were expressed in form of frequency (percentage). $P$ value was significant if $<0.05{ }^{*}$ ).

As regards type of response there was a statistically significant difference between Nilotinib and Imatinib group with favoring Nilotinib with $P=0.01$.

Optimal response occurred in $12(48 \%)$ and $11(44 \%)$ patients in Nilotinib and Imatinib groups respectively while early molecular response occurred in 8 (32\%) and 7 (28\%) patients respectively. Five (20\%) and 7 (28\%) patients in $\mathrm{Ni}$ lotinib and Imatinib group respectively had warning response.

\section{- At 1 the $18^{\text {th }}$ of therapy}

Regarding the major molecular response (MMR) at the $18^{\text {th }}$ month of therapy, Nilotinib group had significantly higher frequency of MMR where 22 (88\%) patients achieved it while only16 (64\%) patients in Imatinib group achieved MMR $(P=0.02)$.

As regards type of response there was a statistically significant difference between Nilotinib and Imatinib group with favoring Nilotinib with $P=0.02$

Optimal response occurred in $12(48 \%)$ and $9(36 \%)$ patients in Nilotinib and Imatinib groups respectively while early molecular response occurred in 10 $(40 \%)$ and 7 (28\%) patients respectively. Three (12\%) and 9 (36\%) patients in Nilotinib and Imatinib group respectively had warning response.

- At the end of study 24 months 
Regarding the major molecular response (MMR), the current study showed that Nilotinib group had significantly higher frequency of MMR where 23 (92\%) patients achieved it while only $16(64 \%)$ patients in Imatinib group achieved $\operatorname{MMR}(P=0.01)$.

As regards type of response there was a statistically significant difference between Nilotinib and Imatinib group with favoring Nilotinib with $P=0.04$

Optimal response occurred in 11 (44\%) and 6 (24\%) patients in Nilotinib and Imatinib groups respectively while early molecular response occurred in 12 (48\%) and $10(40 \%)$ patients respectively. Two (8\%) and $9(36 \%)$ patients in $\mathrm{Ni}$ lotinib and Imatinib group respectively had warning response.

Hematological adverse effects in both groups:

Anemia was considered if $\mathrm{Hb}$ level below $10 \mathrm{mg} \%$. Three (12\%) and 5 (20\%) patients in Nilotinib and Imatinib group respectively developed anemia $(P=$ $0.13)$.

Leucopenia (TLC $<3 \times 10^{3} / \mathrm{ml}$ ) occurred in three $(12 \%)$ patients in each group $(P=0.99)$. None of those received Nilotinib developed thrombocytopenia (platelets $\left.<100 \times 10^{3} / \mathrm{ml}\right)$ while $2(8 \%)$ patients in Imatinib group developed $(P$ $=0.22)$ (Table 3$)$.

Frequency of hepatic impairment and renal impairment in both groups:

It was noticed that frequency of liver cell Impariement (LCI) and renal impaired (RI) was statistically higher in the Imatinib group in comparison to Nilotinib group $(2(8 \%)$ versus $10(40 \%)$ patients; $P=0.02$ for liver cell impairment and $2(8 \%)$ versus $3(12 \%)$ patients; $P=0.03)$. so both were significant (Table 4 ).

Non hematological adverse events:

Grade 1 and 2 myalgia occurred in 7 (28\%) and 2 (8\%) patients from Nilotinib group respectively and occurred in $6(24 \%)$ and $7(28 \%)$ patients from Imatinib group respectively with no significant difference between both groups $(P=0.18)$ (Table 5, Figure 2).

Table 3. Hematological adverse effects of therapy in both groups.

\begin{tabular}{cccc}
\hline Adverse effects & $\begin{array}{c}\text { Nilotinib group } \\
(\mathbf{n}=\mathbf{2 5})\end{array}$ & $\begin{array}{c}\text { Imatinib group } \\
(\mathrm{n}=25)\end{array}$ & $\boldsymbol{P \text { value }}$ \\
\hline Anemia & $3(12)$ & $5(20)$ & 0.13 \\
Leucopenia & $3(12)$ & $3(12)$ & 0.99 \\
Thrombocytopenia & 0 & $2(8)$ & 0.22 \\
\hline
\end{tabular}

Data was expressed in form of frequency (percentage). $P$ value was significant if $<0.05\left(^{*}\right)$.

Table 4. Frequency of hepatic and renal impairment.

\begin{tabular}{cccc}
\hline Adverse effects & $\begin{array}{c}\text { Nilotinib group } \\
(\mathbf{n}=\mathbf{2 5})\end{array}$ & $\begin{array}{c}\text { Imatinib group } \\
(\mathbf{n}=25)\end{array}$ & $\boldsymbol{P}$ value \\
\hline Hepatic impairment & $2(8)$ & $10(40)$ & $0.02^{\star}$ \\
Renal impairment & $2(8)$ & $3(12)$ & $0.03^{\star}$ \\
\hline
\end{tabular}

Data was expressed in form of frequency (percentage). $P$ value was significant if $<0.05\left(^{*}\right)$. 


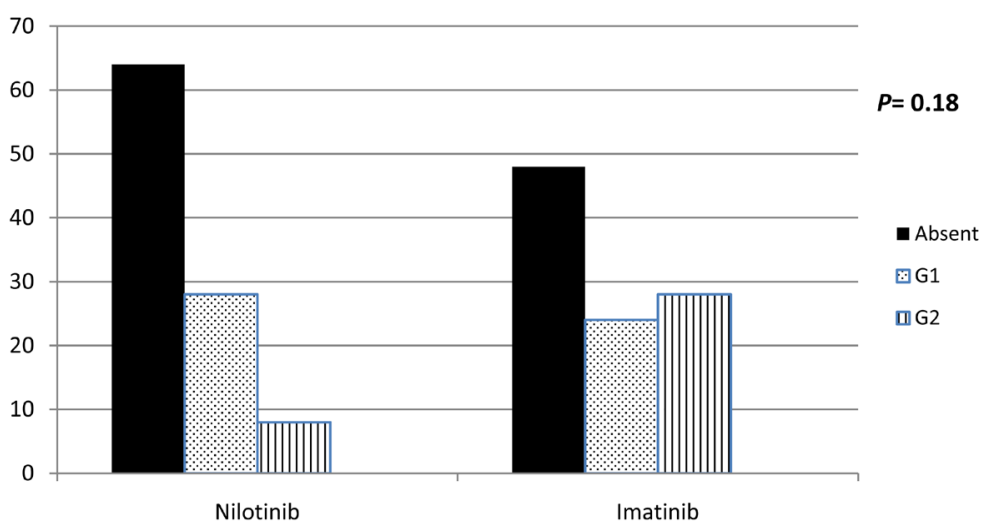

Figure 2. Frequency of myalgia in both groups where data was expressed in form of percentage.

Table 5. Non haematological adverse effects of therapy in both groups during course of therapy.

\begin{tabular}{|c|c|c|c|}
\hline Adverse effects & $\begin{array}{l}\text { Nilotinib group } \\
\quad(\mathrm{n}=25)\end{array}$ & $\begin{array}{l}\text { Imatinib group } \\
\quad(\mathrm{n}=25)\end{array}$ & $P$ value \\
\hline \multicolumn{4}{|l|}{ Myalgia } \\
\hline Absent & $16(64)$ & $12(48)$ & \multirow{3}{*}{0.18} \\
\hline G1 & $7(28)$ & $6(24)$ & \\
\hline G2 & $2(8)$ & $7(28)$ & \\
\hline \multicolumn{4}{|l|}{ Rash } \\
\hline Absent & $25(100)$ & $14(56)$ & \multirow{4}{*}{$0.01^{*}$} \\
\hline G1 & 0 & $6(24)$ & \\
\hline G2 & 0 & $2(8)$ & \\
\hline G3 & 0 & $3(12)$ & \\
\hline \multicolumn{4}{|l|}{ Nausea } \\
\hline Absent & $17(68)$ & $10(40)$ & \multirow{3}{*}{$0.04^{*}$} \\
\hline G1 & $8(32)$ & $11(44)$ & \\
\hline G2 & 0 & $4(16)$ & \\
\hline \multicolumn{4}{|l|}{ Fatigue } \\
\hline Absent & $17(68)$ & $8(32)$ & \multirow{3}{*}{$0.01^{*}$} \\
\hline G1 & $5(20)$ & $15(60)$ & \\
\hline G2 & $3(12)$ & $2(8)$ & \\
\hline \multicolumn{4}{|l|}{ Diarrhea } \\
\hline Absent & $14(56)$ & $18(72)$ & \multirow{3}{*}{0.19} \\
\hline G1 & $8(32)$ & $7(28)$ & \\
\hline G2 & $3(12)$ & 0 & \\
\hline \multicolumn{4}{|l|}{ Fluid retention } \\
\hline Absent & $25(100)$ & $13(42)$ & \multirow{3}{*}{$0.01^{*}$} \\
\hline G1 & 0 & $8(32)$ & \\
\hline G2 & 0 & $4(16)$ & \\
\hline
\end{tabular}

Data was expressed in form of frequency (percentage). $P$ value was significant if $<0.05$. 
None of Nilotinib group developed rash while 6 (24\%), 2 (8\%) and 3 (12\%) patients from Imatinib group developed G1, G2 and G3 rash respectively with significant difference between both groups $(P=0.01)$ (Table 5, Figure 3 ).

Frequency of fatigue was significantly higher $(P=0.01)$ in Imatinib group where G1 and G2 fatigue occurred in $15(60 \%)$ and $2(8 \%)$ patients respectively. Five (20\%) and 3 (12\%) patients from Nilotinib group had G1 and G2 Fatigue (Table 5, Figure 4).

Imatinib group had higher frequency of nausea in comparison to Nilotinib group $(P=0.04)$. Eleven $(44 \%)$ and $4(16 \%)$ patients from those received Imatinib developed G1 and G2 nausea respectively while only 8 (32\%) patients from Nilotinib group had G1 nausea.it was significant $(p=0.04)$ (Table 5, Figure 5).

In contrast, frequency of diarrhea was higher in Nilotinib group; a total of 8 (32\%) and $3(12 \%)$ patients had G1 and G2 diarrhea respectively while only 7 $(28 \%)$ patients from Imatinib group had G1 diarrhea but this there was insignificant difference between both groups regarding occurrence of diarrhea $(P=$ 0.19) (Table 5, Figure 6).

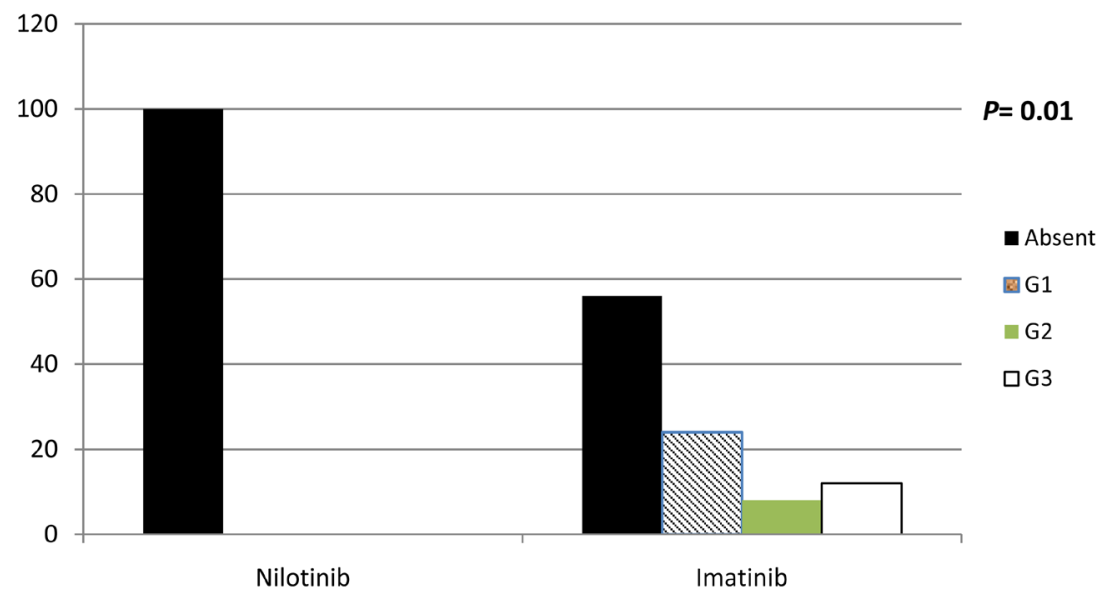

Figure 3. Frequency of rash in both groups where data was expressed in form of percentage.

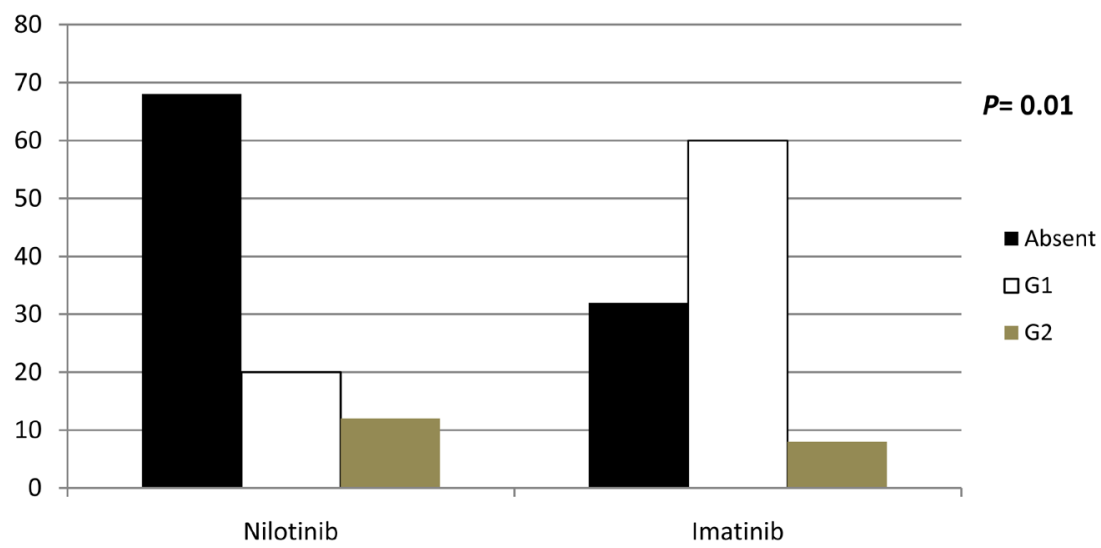

Figure 4. Frequency of fatigue in both groups where data was expressed in form of percentage. 


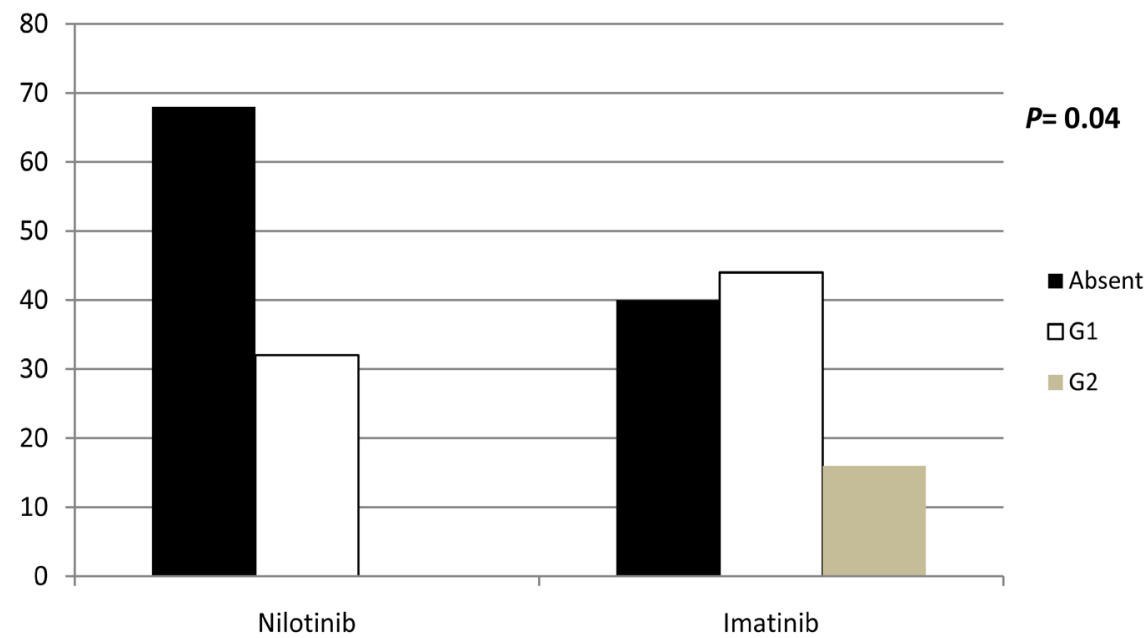

Figure 5. Frequency of nausea in both groups where data was expressed in form of percentage.

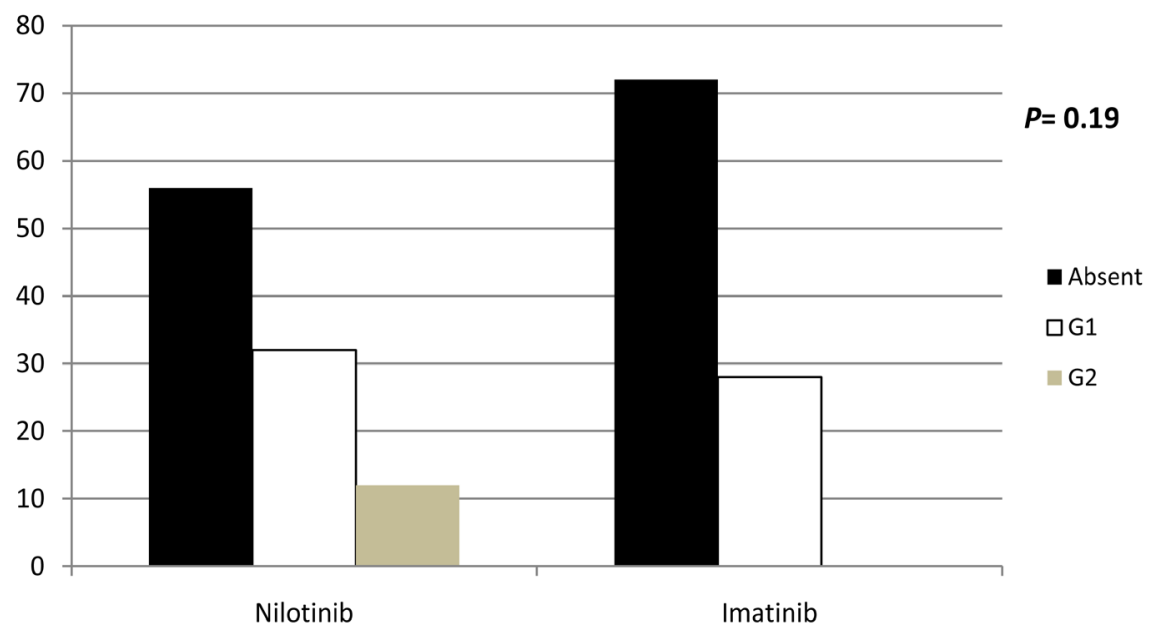

Figure 6. Frequency of diarrhea in both groups where data were expressed in form of percentage.

Fluid retention was noticed only in Imatinib group where $8(32 \%)$ and 4 (16\%) patients had G1 and G2 fluid retention respectively $(P=0.01)$.

Survival analysis in the current study: (Was done by Kaplan Meier test)

Progression free survival: median of disease free progression was 23.44 months in case of Nilotinib group (97.6\%) patients with median of disease free progression was 19.2 ( $80 \%)$ months $(P=0.01)$ (Figure 7).

Median survival analysis: Median survival in the current for nilotinib group was significantly higher than Imatinib group (30 months versus 25 months for Nilotinib group and imatinib group respectively; $P=0.01$ ) (Figure 8).

\section{Duration of response after MMR}

Mean duration of response after MMR was significantly higher in Nilotinib group (19 months) in comparison to Imatinib group (16 months) with $P=0.03$ (Figure 9). 


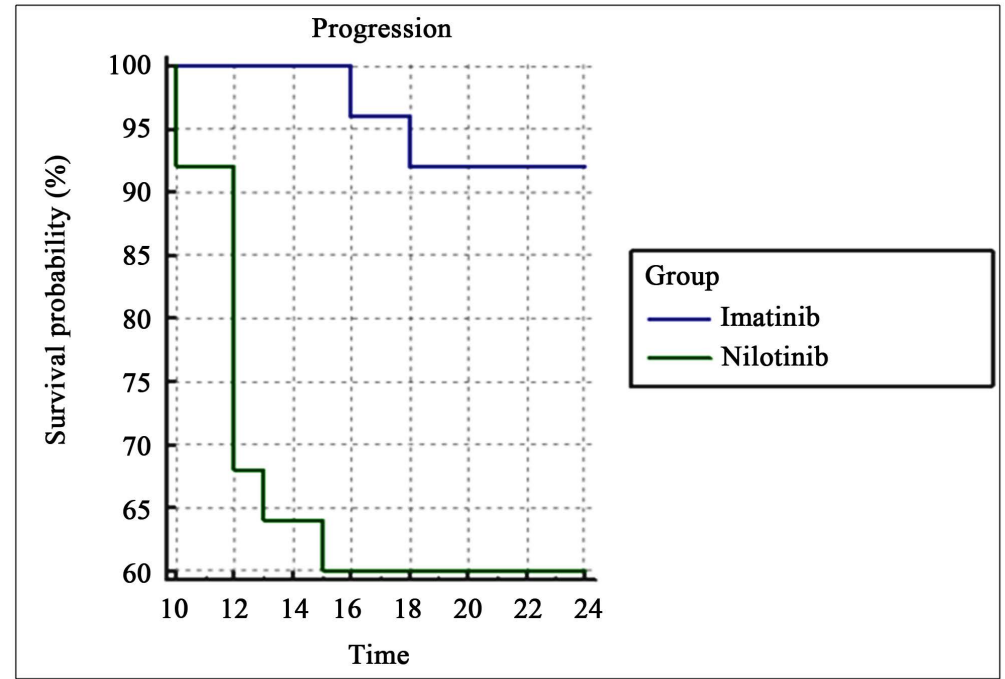

Figure 7. Progression free survival analysis.

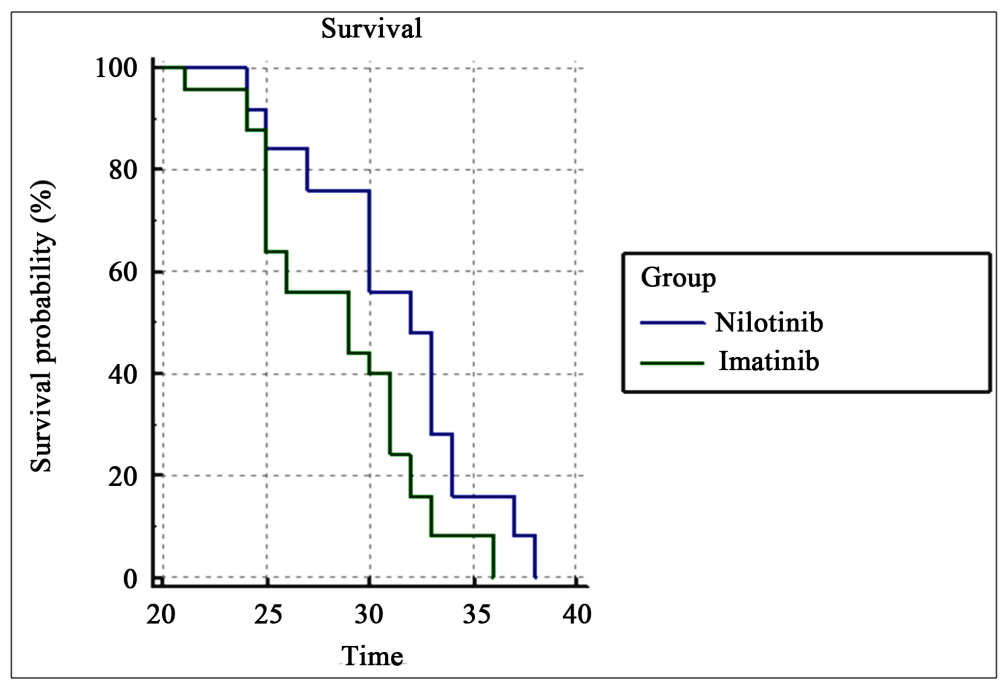

Figure 8. Median survival analysis.

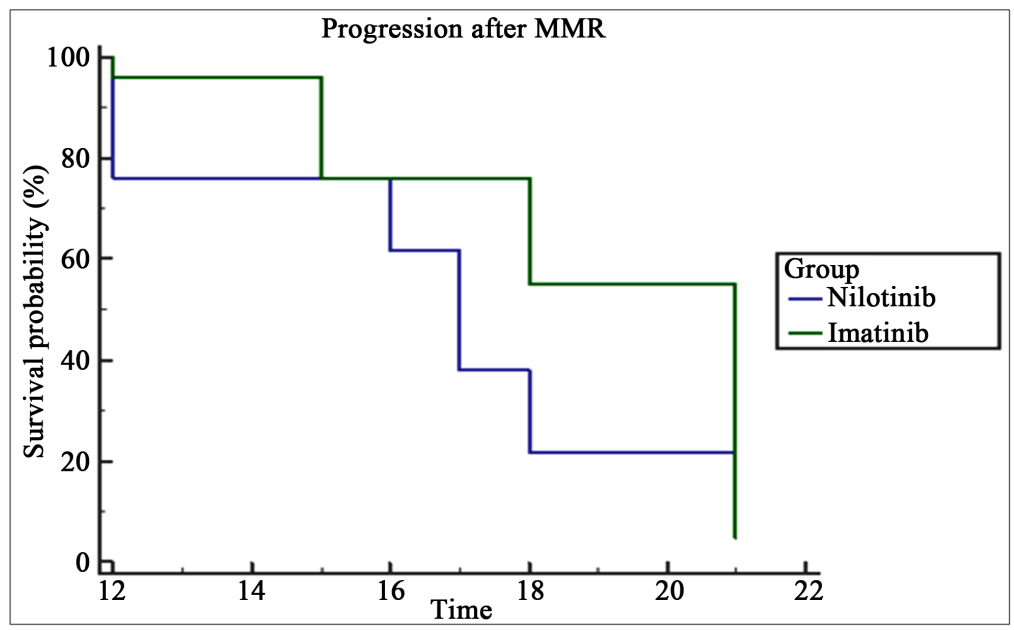

Figure 9. Duration of response after MMR. 


\section{Discussion}

Our study was a 24-months, randomized, phase 3 study designed to compare high dose imatinib (800 mg bid) with nilitonib $400 \mathrm{mg}$ bid in patients with chronic myeloid leukemia who had suboptimal response.

All of the twenty-five patients participating in each group (Nilotinib $800 \mathrm{mg}$ group, and Imatinib $400 \mathrm{mg}$ group) have completed the 24-months period of treatment in the study. No patients in either arm progressed to accelerated phase/blast crisis by 24 months. No deaths or missing during period of study.

Our study showed that Nilotinib group had significantly higher frequency of MMR at $12^{\text {th }}$ month of therapy where $18(72 \%)$ patients achieved it while only 16 (64\%) patients in Imatinib group achieved it $(P=0.04)$. This is similar to a study done by Goh et al. where MMR at $12^{\text {th }}$ month was significantly higher in nilotinib arm compared to imatinib dose-escalation arm $7 / 13(53.85 \%)$ and $2 / 11$ (18.18\%) [8].

At 24 months MMR were 23 (92\%) in Nilotinib arm versus 16 (64\%) in Imatinib arm Also the same results were obtained by Choi and his colleagues in their study where over 3 years the MMR was higher in Nilotinib group than in Imatinib group ( $81.0 \%$ vs $64.6 \%$ in groups 1 , and 2 respectively) $(P=0.01)$ [9].

Moreover, García-Gutiérrez et al. found in their study that MMR by 24 months in Nilotinib group was 64\%, and in Imatinib group 43\% [10].

Contradicting that, Bang and his colleagues in 2017 found that MMR was not significantly different in between treatment arms (41\% vs $29 \%$ of patients) [11].

Regarding types of response, in our study, early molecular response within 3 to 6 months occurred in 12 patients $(48 \%)$ of Nilotinib arm and 10 patients $(40 \%)$ in imatinib arm. Agreeing with us, Koh et al. documented in their study the occurrence of early molecular response within 6 months in $40 \%$ of patients receiving $800 \mathrm{mg}$ Imatinib [12].

On the contrary, Goh et al. have reported that early molecular response had occurred only in patients with nilotinib. [8] Moreover, Ail Awadhi and colleagues documented that $59 \%$ of patients received Nilotinib achieved early molecular response within 3 months [5].

Optimal response, in our study, occurred within 9 to 12 months in 11 (44\%) and $6(24 \%)$ patients in Nilotinib and Imatinib groups, respectively. This is similar to the findings of García-Gutiérrez and colleagues, where after 12 months $42 \%$ of patients achieved optimal response in Nilotinib versus $24 \%$ in Imatinib arm [10].

Similarly, Choi et al. reported the occurrence of optimal response after 12 months in $35 \%$ of patients who received Nilotinib versus only $28 \%$ of those who received Imatinib [9]. Remarkably, Ail Awadhi et al. showed that nine of 12 (75\%) patients who received Nilotinib achieved MMR at 12 months [5].

In our study, only $2(8 \%)$ and $9(36 \%)$ patients in Nilotinib and Imatinib groups respectively had warning response. Our results are the same and slightly better than those obtained by Hughes et al. where 84 (30\%), and 128 (45\%) patients had warning response in nilotinib (400 $\mathrm{mg}$ twice daily), and imatinib 
groups, respectively [13].

Regarding hematologiclal adverse events, anemia occurred in three patients (12\%) receiving Nilotinib and 5 patients (20\%) in Imatinib group respectively $(P$ $=0.13)$. This is better than results obtained by Wang et al. in their study where anemia had occurred in 51 (38.3\%) patients in Nilotinib arm versus 70 (53.0\%) in imatinib [14].

Regarding thrombocytopenia, in our study, none of those received Nilotinib developed thrombocytopenia, while $8(32 \%)$ patients in Imatinib group developed thrombocytopenia $(P=0.22)$. These results are better than results of Saglio et al. where $136(49 \%)$ of patients who received Nilotinib and 156 (56\%) of patients who received imatinib developed thrombocytopenia [15].

Leucopenia was the same in both groups (3 (12\%) in each group $P=0.99)$ in contrary to the results of Larson et al., where leucopenia was significantly higher in Imatinib group with 60 patients (21.4\%) developed it in comparison to only 30 patients (10.8\%) in Nilotinib group [16].

Regarding non-hematological adverse events in our study, renal impairment in the form of increased creatinine level was higher in the Imatinib group in comparison to Nilotinib group (2 (8\%) versus $3(12 \%)$ patients, respectively) $P=$ 0.03 Furthermore, no patients developed acute renal failure.

This is better than results obtained by [17] 7\% of patients developed ac acute kidney injury; creatinine levels returned to baseline in only one of them in patients taking high dose imatinib [17].

Imatinib group had higher frequency of nausea in comparison to Nilotinib group $(P=0.04)$. Eleven $(44 \%)$ and $4(16 \%)$ patients from those received Imatinib developed G1 and G2 nausea respectively while only 8 (32\%) patients from Nilotinib group had G1 nausea these results are better than obtained by Deininger et al. that doucomented G3 and G4 in 2 (3\%) patients received high dose imatinib $800 \mathrm{mg}$ [18].

Also Kantarjian et al. documented G3 and G4 in $3(<1 \%)$ patients received high dose Nilotinib $800 \mathrm{mg}$ [19].

Regarding diahrrea was higher in Nilotinib group; a total of 8 (32\%) and 3 (12\%) patients had G1 and G2 diarrhea respectively while only 7 (28\%) patients from Imatinib group had G1 diarrhea but this there was insignificant difference between both groups regarding occurrence of diarrhea $(P=0.19)$. but in contrary results had occurred with Saglio et al., no diarrhea had occur with Nilotinib arm and only 3(1\%) in. Imatinib arm [15].

Frequency of fatigue was significantly higher $(P=0.01)$ in Imatinib group where G1 and G2 fatigue occurred in $15(60 \%)$ and $2(8 \%)$ patients respectively. Five (20\%) and 3 (12\%) patients from Nilotinib group had G1 and G2 Fatigue. In contrary with study had done by Saglio et al. that showed Frequency of fatigue was significantly higher in nilotinib group $25(9 \%)$. Versus $22(8 \%)$ in imatinib arm with G3 and G4 had occurred more common with Nilotinib group 2 (1\%) versus 1 (less than $1 \%$ ) [15].

Grade 1 and 2 myalgia occurred in $7(28 \%)$ and $2(8 \%)$ patients from Nilotinib 
group respectively and occurred in $6(24 \%)$ and 7 (28\%) patients from Imatinib group respectively with no significant difference between both groups $(P=0.18)$.

On the contrary, Saglio et al. found the incidence of myalgia appears roughly the same with nilotinib and imatinib both 28 (10\%) [15].

None of Nilotinib group developed rash while 6 (24\%), 2 (8\%) and 3 (12\%) patients from Imatinib group developed G1, G2 and G3 rash respectively with significant difference between both groups $(P=0.01)$.in contrary to Wang et al. study they found that patients in Nilotinib group developed rash 47 (35.3\%) and $2(1.5 \%)$ were Grade $3 / 4$ while in Imatinib group rash is less its percentage 17 (12.9\%) and 1 (0.8) [14].

Regarding fluid retention in our study we noticed that it occurred only in Imatinib group $8(32 \%)$ and $4(16 \%)$ patients had G1 and G2 fluid retention respectively $(P=0.01)$.

But Wang et al. documented its occurrence in both groups and only grade land 2 are detected $4(3.0 \%)$ in Nilotinib group versus $23(17.4 \%)$ in Imatinib group [14].

Our survival analysis By using Kaplan Meier survival analysis:

The estimated rate of median survival for Nilotinib group was significantly higher than Imatinib group (30 months versus 25 months for Nilotinib group and Imatinib group, respectively) this is consisted with study by Wang et al. showed median survival arm was 22.3 months in the nilotinib arm and 22.6 months in the imatinib arm [14]. Unfortunately, we could not calculate the overall survival, as we had no deaths among our patients.

The estimated progression free survival rate occurred in Nilotinib a median of disease free progression of 23.44 months (97.6\%) in case of Nilotinib group, while in case of Imatinib group with a median of disease free progression of 19.2 ( $80 \%)$ months.

This is in contrary to García-Gutiérrez et al. that found PFS rate in both groups were similar 92.6\% [10]. Also Wang et al. showed PFS in both groups was $95.6 \%$ [14].

Finally, mean duration of response after MMR was significantly higher in Nilontinib group (19 months) than in Imatinib group (16 months). This is nearly similar to a study done by Ishikawa and his colleagues that showed duration of response after MMR 26 months in Nilontinib group versus 23.8 months in Imatinib group [20].

\section{Conclusion}

In conclusion, both Nilotinib and high dose Imatinib achieved response in CML patients with suboptimal response with rapid and deeper molecular response, better survival outcomes and less side effects in nilotinib.

\section{Conflicts of Interest}

The authors declare no conflicts of interest regarding the publication of this paper. 


\section{References}

[1] Jabbour, E. and Kantarjian, H. (2017) Chronic Myeloid Leukemia: 2018 Update on Diagnosis, Monitoring, and Management. Annual Clinical Updates in Haematological Malignancies.

[2] Soverini, S., Hochhaus, A., Nicolini, F.E., et al. (2011) BCR-ABL Kinase Domain Mutation Analysis in Chronic Myeloid Leukemia Patients Treated with Tyrosine Kinase Inhibitors: Recommendations from an Expert Panel on Behalf of European LeukemiaNet. Blood, 118, 1208-1215. https://doi.org/10.1182/blood-2010-12-326405

[3] National Comprehensive Cancer Network (NCCN) (2016) Clinical Practice Guidelines in Oncology. Chronic Myelogenous Leukemia.

[4] Baccarani, M., Castagnetti, F., Gugliotta, G., et al. (2014) Treatment Recommendations for Chronic Myeloid Leukemia Mediterr. Mediterranean Journal of Hematology and Infectious Diseases, 6, e2014005.

[5] Ailawadhi, S., Luke, P.A., Carole, B.M., et al. (2017) Exploratory Study on the Impact of Switching to Nilotinib in 18 Patients with Chronic Myeloid Leukemia in Chronic Phase with Suboptimal Response to Imatinib. Therapeutic Advances in Hematology, 8, 3-12. https://doi.org/10.1177/2040620716678118

[6] Hochhaus, A., Saglio, G., Hughes, T.P., et al. (2016) Long-Term Benefits and Risks of Frontline Nilotinib vs Imatinib for Chronic Myeloid Leukemia in Chronic Phase: 5-Year Update of the Randomized ENESTnd Trial. Leukemia, 30, 1044-1054. https://doi.org/10.1038/leu.2016.5

[7] Baccarani, M., Deininger, M., Rosti, A., et al. (2013) European LeukemiaNet Recommendations for the Management of Chronic Myeloid Leukemia. Blood, 122, 885-892. https://doi.org/10.1182/blood-2013-05-501569

[8] Goh, H.G., Jootar, S., Kim, H.J., et al. (2011) Efficacy of Nilotinib versus High-Dose Imatinib in Early Chronic Phase CML Patients Who Have Suboptimal Molecular Responses to Standard-Dose Imatinib (RE-NICE Multicenter Study) Blood. (ASH Annual Meeting Abstracts). Vol. 118. Abstract 2765.

[9] Choi, S.Y., Lee, S.E., Kim, S.H., et al. (2015) Efficacy of Nilotinib versus High Dose Imatinib versus in Early Chronic Phase CML Patients Who Have Suboptimal Response to Front Line Imatinib. EHA Learning Center, Cancer Research Institute.

[10] García-Gutiérrez, V., Puerta, J.M., Maestro, B., et al. (2014) Do Chronic Myeloid Leukemia Patients with Late "Warning" Responses Benefit from "Watch and Wait" or Switching Therapy to a Second Generation Tyrosine Kinase Inhibitor? American Journal of Hematology, 89, E206-E211. https://doi.org/10.1002/ajh.23816

[11] Bang, J.-H., Byeun, J.-Y., Choi, S.-Y., et al. A Phase III Multi-Center, Open-Label, Randomized Study of the Efficacy of Nilotinib versus Imatinib in Adult Patients With Ph+ CML in Early CP Who Have a Suboptimal Molecular Response to Imatinib. ClinicalTrials.gov: US National Institutes of Health NCT01400074 Latest Information Update 2017.

[12] Koh, Y., Lee, J.H. and Lee, K.H. (2010) Phase IV Study Evaluating Efficacy of Escalated Dose of Imatinib in Chronic Myeloid Leukemia Patients Showing Suboptimal Response to Standard Dose Imatinib. Annals of Hematology, 89, 725-731. https://doi.org/10.1007/s00277-010-0910-8

[13] Hughes, T., Hochhaus, A., Kantarjian, H., et al. (2014) Safety and Efficacy of Switching to Nilotinib $400 \mathrm{mg}$ Twice Daily for Patients with Chronic Myeloid Leukemia in Chronic Phase with Suboptimal Response or Failure on Frontline Imatinib or Nilotinib 300 mg Twice Daily. Haematologica, 99, 1204. 
https://doi.org/10.3324/haematol.2013.091272

[14] Wang, J., Shen, Z.X., Saglio, G., Jin, J., Huang, H., Hu, Y., et al. (2015) Phase 3 Study of Nilotinib vs Imatinib in Chinese Patients with Newly Diagnosed Chronic Myeloid Leukemia in Chronic Phase: ENESTchina. Blood, 125, 2771-2778.

https://doi.org/10.1182/blood-2014-09-601674

[15] Saglio, G., Kim, D.W., Issaragrisil, S., et al. (2010) Nilotinib versus Imatinib for Newly Diagnosed Chronic Myeloid Leukemia. The New England Journal of Medicine, 362, 2251-2259. https://doi.org/10.1056/NEJMoa0912614

[16] Larson, R.A., Hochhaus, A., Hughes, T.P., et al. (2012) Nilotinib vs Imatinib in Patients with Newly Diagnosed Philadelphia Chromosome-Positive Chronic Myeloid Leukemia in Chronic Phase: ENESTnd 3-Year Follow-Up. Leukemia, 26, 2197-2203. https://doi.org/10.1038/leu.2012.134

[17] Marcolino, M.S., Boersma, E. and Clementino, N.C.D. (2011) Imatinib Treatment Duration Is Related to Decreased Estimated Glomerular Filtration Rate in Chronic Myeloid Leukemia Patients. Annals of Oncology, 22, 2073-2079.

https://doi.org/10.1093/annonc/mdq715

[18] Deininger, M.W., Kopecky, K.J. and Radich, J.P. (2013) Imatinib 800 mg Daily Induces deeper Molecular Responses than Imatinib 400 mg Daily: Results of SWOG S0325, an Intergroup Randomized PHASE II Trial in Newly Diagnosed Chronic Phase Chronic Myeloid Leukaemia. British Journal of Haematology, 164, 223-232. https://doi.org/10.1111/bjh.12618

[19] Kantarjian, H.M., Giles, F.J., Bhalla, K.N., et al. (2012) Nilotinib Is Effective in Patients with Chronic Myeloid Leukemia in Chronic Phase after Imatinib Resistance or Intolerance: 24-Month Follow-Up Results. Blood, 117, 1141-1145. https://doi.org/10.1182/blood-2010-03-277152

[20] Ishikawa, J., Matsumura, I., Kawaguchi, T., et al. (2018) Efficacy and Safety of Switching to Nilotinib in Patients with CML-CP in Major Molecular Response to Imatinib: Results of a Multicenter Phase II Trial (NILSw Trial). International Journal of Hematology, 107, 535-540. https://doi.org/10.1007/s12185-018-2401-y 